\title{
A Spectral Model for RF Oscillators With Power-Law Phase Noise
}

\author{
Arsenia Chorti and Mike Brookes, Member, IEEE
}

\begin{abstract}
In this paper, we apply correlation theory methods to obtain a model for the near-carrier oscillator power-spectral density (PSD). Based on the measurement-driven representation of phase noise as a sum of power-law processes, we evaluate closed form expressions for the relevant oscillator autocorrelation functions. These expressions form the basis of an enhanced oscillator spectral model that has a Gaussian PSD at near-carrier frequencies followed by a sequence of power-law regions. New results for the effect of white phase noise, flicker phase noise and random walk frequency modulated phase noise on the near-carrier oscillator PSD are derived. In particular, in the case of 1 / $f$ phase noise, we show that despite its lack of stationarity it is possible to derive a closed form expression for its effect on an oscillator PSD and show that the oscillator output can be considered to be wide-sense stationary.
\end{abstract}

Index Terms-Correlation theory, frequency noise, Gaussian PSD, Lorentzian PSD, oscillator power-spectral density (PSD), phase noise, power-law process.

\section{INTRODUCTION}

$\mathbf{I}$ N RADIO frequency communications, oscillatory systems play a prominent role as they allow the practical realization of important operations including frequency translation, synchronization, timing, and filtering. Because of their importance, the analysis of noise in real oscillators has been the subject of much research.

A number of models have been proposed for characterizing the power-spectral density (PSD) of oscillators based on knowledge of the phase noise spectral characteristics. Amongst them, the Leeson model [1] remains probably the most widely used. Recent work by Hajimiri and Lee [2] introduced a new linear time-variant approach and considered the transformation of noise in higher order harmonics into near-carrier phase noise. In addition, Demir et al. in [3] have developed an elaborate time-domain analysis and numerical methods for the characterization of phase noise and discuss the oscillator PSD shape when the phase noise is generated from white frequency noise.

Empirical models based on measurements suggest that the phase noise PSD can be described as a sum of power-law processes $k_{\alpha}|f|^{-\alpha}$ with $\alpha \in\{0,1,2,3,4\}$ [4], [5]. The model for

Manuscript received July 19, 2005; revised December 9, 2005. This work was supported by Panasonic System LSI Design Europe. This paper was recommended by Associate Editor V. E. DeBrunner.

A. Chorti is with the Department of Electronics and Computer Science, University of Southampton, SO17 1BJ, U.K. (e-mail: ac2@ecs.soton.ac.uk, ersi. chorti@gmail.com).

M. Brookes is with the Department of Electrical and Electronic Engineering, Imperial College London, London SW7 2AZ, U.K. (e-mail: mike.brookes@ imperial.ac.uk).

Digital Object Identifier 10.1109/TCSI.2006.881182
TABLE I

NOISE COLORS

\begin{tabular}{ll}
\hline$\omega^{-\alpha}$ Phase Noise & Color of $\Omega(t)$ \\
\hline \hline$\omega^{0}:$ White Phase Noise & Purple \\
$\omega^{-1}:$ Flicker Phase Noise & Blue \\
$\omega^{-2}:$ White FM Phase Noise & White \\
$\omega^{-3}:$ Flicker FM Phase Noise & Pink \\
$\omega^{-4}:$ Random Walk FM Phase Noise & Brown \\
\hline
\end{tabular}

the phase noise generation mechanism is based on the transformation of frequency fluctuations expressed in the Laplace domain as $\Omega(s)$, to phase fluctuations

$$
\Phi(s)=\frac{\Omega(s)}{s} .
$$

This leads to a spectral transformation of the type

$$
S_{\phi}(\omega)=\frac{S_{\Omega}(\omega)}{\omega^{2}}
$$

where $S_{\phi}$ and $S_{\Omega}$ are the phase noise PSD and frequency noise PSD, respectively [6]. In this paper, we adopt the widely used convention of referring to various noise sources as "colored." The correspondence between the terminology for frequency noise sources and the resulting phase noise is summarized in Table I.

According to the above model, white phase noise is generated by purple frequency noise with PSD proportional to $f^{2}$ while flicker phase noise is generated by blue frequency noise with PSD proportional to $|f|$. This representation does not correspond to the actual phase noise generation mechanisms in those two cases and only serves as a mathematical abstraction. In reality, we do not expect white phase noise to be generated by purple frequency noise or flicker phase noise to be generated by blue frequency noise but rather, as Leeson suggested in [1], we expect that white and flicker noise sources are phase modulated in the oscillator loop. Suggestions concerning the time-domain modeling of these processes can be found in [7]-[9].

For $\alpha \geq 1$, the power-law spectra have infinite power at low frequencies and therefore cannot provide an accurate model for the near-carrier PSD of a real oscillator. So far, the question of near-carrier oscillator PSD has been successfully addressed for the case of white frequency noise [10], and the oscillator PSD in this case is established as a Lorentzian. ${ }^{1}$ The result is a straightforward consequence of the analysis in [11] and was confirmed by Demir et al. in [3]. Klimovitch in [12] and [13] has

\footnotetext{
${ }^{1}$ Lorentzian is the shape of the power spectrum of a first-order low-pass filter.
} 
proposed a new analysis for the case of pink frequency noise and predicts a Gaussian² PSD close to the oscillator frequency.

The aim of this paper is to determine the near-carrier oscillator PSD by applying the methodology presented in [10], [11], and [12] for the power-law phase noise processes described in Table I. In our approach, we apply correlation theory techniques to derive closed form expressions for the oscillator autocorrelation function. Novel analyses and results are presented for the near-carrier regime in the cases of purple, blue, and brown frequency noise that avoid the infinite power difficulties mentioned above. Our findings for the near-carrier PSD are combined in an enhanced oscillator spectral model that accounts for all the main types of power-law phase noise.

This paper is organized as follows. In Section II, we provide the theoretical background on phase noise and the correlation theory of frequency fluctuations. In Sections III to VII, the cases of purple, blue, white, pink and brown frequency noise are examined individually. In Section VIII, we combine these to give an overall oscillator spectral model and in Section IX, we state our conclusions.

\section{BACKGROUND}

\section{A. Phase Noise and the Small-Angle Approximation}

Noisy oscillators are described in the time domain as

$$
\zeta(t)=(1+\varepsilon(t)) \cos \left(\omega_{\mathrm{osc}} t+\phi(t)\right)
$$

where $\varepsilon(t)$ and $\phi(t)$ are real random processes (RPs). To isolate the effect of phase noise on the oscillator PSD, we will assume that amplitude fluctuations expressed through $\varepsilon(t)$ have a negligible effect [10]. In support of this assumption, it is shown in [12] that there exists a frequency range close to the nominal oscillator frequency $\omega_{\text {osc }}$ where the effect of amplitude noise is negligible compared to that of phase noise.

In order to simplify the analysis, we will continue considering the complex valued oscillation

$$
\psi(t)=e^{j\left(\omega_{\mathrm{osc}} t+\phi(t)\right)}
$$

where $\psi(t)$ is an analytic version of $\zeta(t)$ assuming $\varepsilon(t)=0$. In Section II-B we note that it is possible to reconstruct the PSD of the real valued oscillator $\zeta(t)$ from the PSD of the complex valued $\psi(t)$ provided that $\zeta(t)$ is wide-sense stationary (WSS).

The effect of $e^{j \phi(t)}$ term in (3) is that the delta function PSD of an ideal oscillator $e^{j \omega_{\text {osc }} t}$ is widened with energy spreading to neighboring frequencies. The oscillator PSD can be derived by frequency translation by $\omega_{\text {osc }}$ of the PSD of the component $e^{j \phi(t)}$. Consequently, in the following, we will only be interested in the PSD of the RP:

$$
w(t)=e^{j \phi(t)} .
$$

It is common in the literature to assume that $\phi(t)$ is a zeromean WSS process and that it is sufficiently small to make the "small-angle approximation:" $w(t)=e^{j \phi(t)} \simeq 1+j \phi(t)$. With this approximation, we obtain

$$
\begin{aligned}
R_{w w}(\tau)= & \left\langle w^{*}(t) w(t+\tau)\right\rangle \\
& \simeq\langle(1-j \phi(t))(1+j \phi(t+\tau))\rangle \\
& =1+R_{\phi \phi}(\tau) \\
& \text { so that } \\
S_{w}(\omega) & \simeq 2 \pi \delta(\omega)+S_{\phi}(\omega)
\end{aligned}
$$

where $\langle\cdot\rangle$ denotes statistical expectation and $R_{w w}, S_{w}$, and $R_{\phi \phi}, S_{\phi}$ represent the autocorrelation and PSD of $w(t)$ expressed in (4) and $\phi(t)$ respectively.

The Wiener-Khinchin theorem thus implies that in the range of frequencies the small-angle approximation is applicable, the oscillator PSD coincides with the phase noise PSD. The same conclusion follows from the more elaborate analysis in [10] for the case of a real-valued oscillator expressed as in (2). However, close to the carrier where energy concentration is relatively high, the small-angle approximation fails to provide a good estimate. This effect of low frequency noise in oscillators is characterized through their long-term stability and can manifest itself through non-negligible drifts in the oscillation frequency.

In the following section, we present the theoretical model for phase noise generation from frequency fluctuations. This approach, which avoids the small-angle approximation, is a useful frequency-domain technique for the determination of the nearcarrier PSD.

\section{B. Correlation Theory of Frequency Fluctuations}

The present section is an extension of the analysis in [10] to the case of complex valued oscillators. Let us assume a unit amplitude oscillator with time response as in (3), where $\phi(t)$ is an RP that we assume to be zero-mean Gaussian (ZMG) with variance $\sigma_{\phi}^{2}(t)$.

Assuming that $w(t)=e^{j \phi(t)}$ is WSS, its autocorrelation function can be expressed as

$$
R_{w w}(\tau)=\left\langle e^{j(\phi(t+\tau)-\phi(t))}\right\rangle \doteq\left\langle e^{j \xi(t, \tau)}\right\rangle
$$

The "phase noise variation" $\operatorname{RP} \xi(t, \tau)=\phi(t+\tau)-\phi(t)$ that appears in the autocorrelation function $R_{w w}(\tau)$ results from the addition of two ZMG RPs and is therefore a zero-mean RP. We can express the PSD of $w(t)$ as the Fourier transform $(\mathcal{F})$ of its autocorrelation function

$$
S_{w}(\omega)=\mathcal{F}\left[R_{w w}(\tau)\right]=\int_{-\infty}^{\infty} R_{w w}(\tau) e^{-j \omega \tau} \mathrm{d} \tau
$$

In order to evaluate (7) and (8), we need some information about the phase noise variation $\operatorname{RP} \xi(t, \tau)$. We will assume that $\phi(t)$ and $\phi(t+\tau)$ are jointly Gaussian and ergodic random variables, so that the probability density function of $\xi(t, \tau)$ can be expressed as

$$
f_{\xi}(\xi ; t, \tau)=\frac{1}{\sqrt{2 \pi} \sigma(\tau)} e^{-\frac{\xi^{2}(t, \tau)}{2 \sigma^{2}(\tau)}}
$$

\footnotetext{
${ }^{2}$ This is also known as Doppler lineshape.
} 


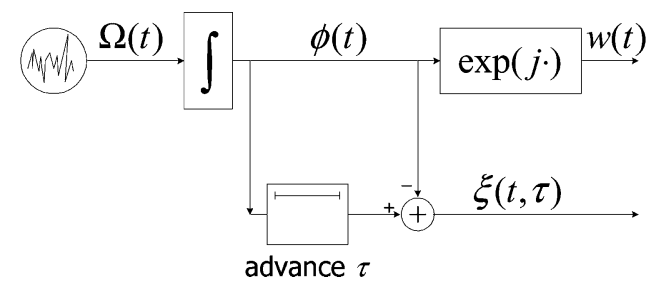

Fig. 1. Phase noise generation from frequency fluctuations.

where $\sigma^{2}(\tau)$ is the variance of the $\operatorname{RP} \xi(t, \tau)$. From (7) and (9) we can evaluate

$$
R_{w w}(\tau)=e^{\frac{-\sigma^{2}(\tau)}{2}}
$$

At this point it, is worth noting that WSS of $w(t)$ does not presuppose WSS of $\phi(t)$ but reduces to the following requirements if $\phi(t)$ and $\phi(t+\tau)$ are jointly Gaussian and ergodic: i) that $\sigma_{\phi}^{2}$ is constant; and ii) $\sigma^{2}(\tau)$ be bounded. The first requirement suggests $w(t)$ has a constant expected value

$$
\langle w(t)\rangle=\left\langle e^{j \phi(t)}\right\rangle=e^{-\frac{\sigma_{\phi}^{2}}{2}}
$$

while the second requirement ensures convergence of $R_{w w}(\tau)$.

The variance $\sigma^{2}(\tau)$ of the process $\xi(t, \tau)$ is called the variogram of $\phi(t)$ and is a quantity extensively used in geostatistics. When the process $\phi(t)$ is zero-mean WSS, its variogram can be evaluated as [14], [15]

$$
\sigma^{2}(\tau)=2\left(R_{\phi \phi}(0)-R_{\phi \phi}(\tau)\right)
$$

Since $R_{\phi \phi}(\tau)$ is an even function of $\tau$, it follows from (10) and (12) that the autocorrelation function of the RP $w(t)$ is a real and even function of $\tau$. Consequently, the PSD $S_{w}(\omega)$ will be a real and even function of $\omega$ and the following simplifications are applicable:

$$
\begin{aligned}
S_{w}(\omega) & =2 \int_{0}^{\infty} R_{w w}(\tau) \cos (\omega \tau) \mathrm{d} \tau \\
R_{w w}(\tau) & =\frac{1}{\pi} \int_{0}^{\infty} S_{w}(\omega) \cos (\omega \tau) \mathrm{d} \omega .
\end{aligned}
$$

According to our model, phase noise $\phi(t)$ emerges by integration of frequency fluctuations $\Omega(t)$

$$
\phi(t)=\int_{0}^{t} \Omega\left(t^{\prime}\right) \mathrm{d} t^{\prime}
$$

and therefore, we can express

$$
\xi(t, \tau)=\phi(t+\tau)-\phi(t)=\int_{t}^{t+\tau} \Omega\left(t^{\prime}\right) \mathrm{d} t^{\prime}
$$

In Fig. 1, the generation mechanism of the RPs $\phi(t), \xi(t, \tau)$, and $w(t)$ from the frequency fluctuations $\Omega(t)$ is illustrated.
The variance of $\xi(t, \tau)$ in terms of the $\operatorname{PSD} S_{\Omega}(\omega)$ of $\Omega(t)$ is then expressed as

$$
\begin{aligned}
\sigma^{2}(\tau)= & \left\langle\xi(t, \tau)^{2}\right\rangle \\
= & \left\langle\int_{t}^{t+\tau} \int_{t}^{t+\tau} \Omega(x) \Omega(y) \mathrm{d} y \mathrm{~d} x\right\rangle \\
= & \int_{t}^{t+\tau} \int_{t}^{t+\tau} R_{\Omega \Omega}(x-y) \mathrm{d} y \mathrm{~d} x \\
= & \frac{1}{\pi} \int_{0}^{\infty} S_{\Omega}(\omega) \\
& \times \int_{t}^{t+\tau} \int_{t}^{t+\tau} \cos (\omega(x-y)) \mathrm{d} y \mathrm{~d} x \mathrm{~d} \omega \\
= & \frac{2}{\pi} \int_{0}^{\infty} S_{\Omega}(\omega) \frac{1-\cos (\omega \tau)}{\omega^{2}} \mathrm{~d} \omega
\end{aligned}
$$

where

$$
R_{\Omega \Omega}(\tau)=\frac{1}{\pi} \int_{0}^{\infty} S_{\Omega}(\omega) \cos (\omega \tau) \mathrm{d} \omega
$$

is the autocorrelation of the frequency fluctuations $\Omega(t)$.

From (8), (10), and (15) it follows that the PSD of $w(t)$ can be expressed as

$$
\begin{aligned}
& S_{w}(\omega)=2 \int_{0}^{\infty} f(\tau) \cos (\omega \tau) \mathrm{d} \tau \\
& \text { where } f(\tau)=\exp \left(-\frac{1}{\pi} \int_{0}^{\infty} S_{\Omega}(\lambda) \frac{1-\cos (\lambda \tau)}{\lambda^{2}} \mathrm{~d} \lambda\right)
\end{aligned}
$$

We have thus concluded that the complex oscillator PSD can be derived by frequency translation by $\omega_{\text {osc }}$ of the PSD of the component $w(t)=e^{j \phi(t)}$ expressed in (17). The PSD of a realvalued oscillator (2) is then given by $0.5 S_{w}(\omega)$ which agrees with the expression obtained in [10].

In the following sections, we derive closed form expressions for the autocorrelation function of $w(t)$ when the PSD of $\phi(t)$ follows a power law process $k_{\alpha}|f|^{-\alpha}$. For convenience, the quantities $\phi(t), \xi(t, \tau), w(t), \zeta(t), \sigma^{2}(\tau), \Omega(t), R_{w w}(\tau)$, and $S_{w}(\omega)$ will be indexed with the value of $\alpha$.

\section{White PHASE NOISE}

We begin by examining the case of white phase noise with $\alpha=0$. To account for the realistic case of band-limited whitelike phase noise, we will construct $\phi_{0}(t)$ by low-pass-filtering white noise. Assuming a first-order filter with cutoff frequency $\omega_{B}$, the PSD of $\phi_{0}(t)$ is expressed as

$$
S_{\phi_{0}}(\omega)=k_{0} \frac{\omega_{B}^{2}}{\omega^{2}+\omega_{B}^{2}} .
$$

From (12) and (18), we obtain

$$
\begin{aligned}
\sigma_{0}^{2}(\tau) & =2\left(R_{\phi \phi_{0}}(0)-R_{\phi \phi_{0}}(\tau)\right) \\
& =\frac{2 k_{0} \omega_{B}^{2}}{\pi} \int_{0}^{\infty} \frac{1-\cos (\omega \tau)}{\omega^{2}+\omega_{B}^{2}} \mathrm{~d} \omega \\
& =k_{0} \omega_{B}\left(1-e^{-\omega_{B}|\tau|}\right) .
\end{aligned}
$$




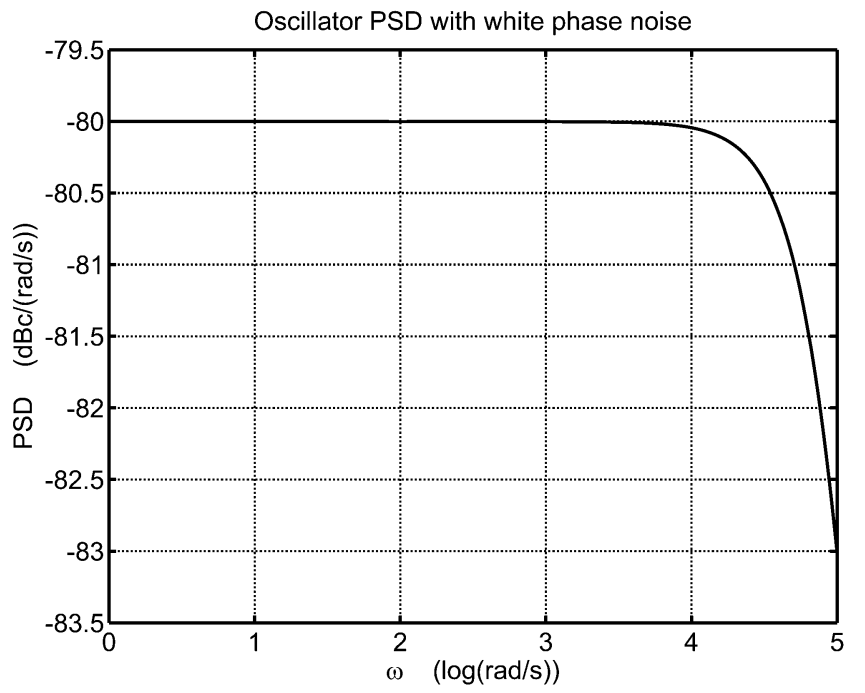

Fig. 2. Phase noise PSD with white noise $k_{0}=10^{-8} \mathrm{~W} / \mathrm{Hz}, \omega_{B}=100$ $\mathrm{krad} / \mathrm{s}$.

As $\tau \rightarrow \infty$, it follows from (19) and (10) that $R_{w w_{0}}(\tau)$ converges to the nonzero value

$$
\lim _{\tau \rightarrow \infty} R_{w w_{0}}(\tau)=e^{-\frac{k_{0} \omega_{B}}{2}} \doteq r_{0} .
$$

In deriving $S_{w_{0}}(\omega)$ from $R_{w w_{0}}(\tau)$ using (8), we can avoid convergence issues and use of limits [16] by treating the component $r_{0}$ separately and expressing $S_{w_{0}}(\omega)$ as

$$
S_{w_{0}}(\omega)=2 \pi r_{0} \delta(\omega)+\mathcal{F}\left[R_{w w_{0}}(\tau)-r_{0}\right]
$$

The component $r_{0}$ of the autocorrelation function $R_{w w_{0}}(\tau)$ corresponds to a tone weighted by $2 \pi r_{0}$ on the carrier frequency while the component $R_{w w_{0}}(\tau)-r_{0}$ characterizes the energy spread in neighboring frequencies. As predicted from the smallangle approximation, the PSD of $w_{0}(t)$ away from the carrier will coincide with the PSD of $\phi_{0}(t)$, which from (1) and (18), has a PSD $k_{0}$ at frequencies smaller than $\omega_{B}$.

In Fig. 2, we plot the numerically evaluated $\mathcal{F}\left[R_{w w_{0}}(\tau)-r_{0}\right]$. The PSD is flat and equals $k_{0}$ as expected while the cutoff frequency is $\omega_{B}$, which was selected in this example as $100 \mathrm{krad} / \mathrm{s}$.

To summarize the preceding analysis, the PSD $S_{w_{0}}(\omega)$ of $w_{0}(t)$ is a Dirac weighted by $2 \pi e^{-\left(k_{0} \omega_{B}\right) /(2)}$ at the carrier frequency plus a component that is flat up to the $3 \mathrm{~dB}$ angular frequency $\omega_{B}$, with PSD $k_{0}$.

\section{FLICKer PhASe NoISE}

It is known that numerous physical fluctuations have empirical (measured) spectral densities proportional to $1 / f^{\alpha}$ with $\alpha$ in the vicinity of $1(\alpha=1 \pm \nu, \nu \ll 1)$. This spectral behavior places two serious controversies in terms of finite power systems analysis. Infinities are inferred, due to both low and high frequencies tendency to approach $\mathrm{dc}^{3}$ and $\infty$ at a $1 / f$ rate.

${ }^{3} \mathrm{DC}$ in terms of this analysis is equivalent to the nominal or average oscillation frequency.
In terms of the high-rate infinity, a widely accepted assumption is that there has to exist a higher frequency $f_{h}$, at which the PSD slope becomes steeper [17]. It has never been possible to measure such a higher corner frequency, because $1 / f$ noise disappears in white thermal noise that is omnipresent in all electronic systems. However, as Plank's analysis for thermal noise revealed a higher cutoff frequency in the case of white noise sources, the above assumption appears plausible.

As a result of the above, research interest was mainly oriented in characterizing the nature of $1 / f$ perturbations near dc. Similarly to the high-frequencies case, the concept of a lower cutoff frequency was introduced, below which the PSD was expected to flatten off. This approach was adopted in [18] for the analysis of $1 / f$ noise sources in the phase of an oscillator. The resulting expression for the oscillator autocorrelation function was used to approximate the $1 / f$ region as a Lorentzian PSD.

Generally, in the past, theoretical models for $1 / f$ processes either proposed its representation as a nonstationary RP that exhibits infinite memory [19], [20], or concluded that the so-called "infrared catastrophe" cannot be tackled with traditional statistical tools [21]. Work by Handel in [22] and [23] examines the origins of $1 / f$ noise in electronic devices in the general context of "quantum $1 / f$ processes." In this work, he argues that the PSD of $1 / f$ processes diverges to a characteristic with a slope smaller that unity near dc, so that the "infrared catastrophe" described in [21] is overcome. Using the theory of quantum $1 / f$ noise, Handel and Tsai in [24] explain the origins of the $1 / f$ region in the PSD of some crystal oscillators.

In this paper, we avoid making the assumption of a lower cutoff frequency. We first consider nonstationary $1 / f$ noise and move to the case of approximately flicker noise with $\alpha \rightarrow 1$.

We are interested in the PSD of $w_{1}(t)=e^{j \phi_{1}(t)}$. Since $\sigma_{\phi}^{2}=$ $\infty, w(t)$ is a zero-mean process. As a result, the stationarity of $w_{1}(t)$ does not require stationarity of $\phi_{1}(t)$ but rather that the variance $\sigma_{1}^{2}(\tau)$ of phase noise variations $\xi_{1}(t, \tau)=\phi_{1}(t+\tau)-$ $\phi_{1}(t)$ be bounded. To produce flicker phase noise, we start with frequency noise $\Omega_{1}(t)$ with an approximate blue noise PSD as

$$
S_{\Omega_{1}}(\omega)=(2 \pi)^{1-\nu} k_{1}\left|\omega^{1-\nu}\right|, \quad \text { with } \nu \rightarrow 0 .
$$

In the case of exactly flicker phase noise with $\nu=0$, we have

$$
\begin{aligned}
\sigma_{1}^{2}(\tau) & =4 k_{1} \int_{0}^{\infty} \frac{1-\cos (\omega \tau)}{\omega} \\
& = \begin{cases}\infty, & \tau \neq 0 \\
\text { undefined, } & \tau=0\end{cases}
\end{aligned}
$$

so that

$$
R_{w w_{1}}(\tau)= \begin{cases}0, & \tau \neq 0 \\ \text { undefined, } & \tau=0\end{cases}
$$

The oscillator output is in this case a nonstationary process in the wide sense. 
In the following, we examine the case of approximately flicker phase noise with $0<\nu \ll 1$. The variance of $\xi_{1}(t, \tau)$ is consequently found from (15) to be

$$
\begin{aligned}
\sigma_{1}^{2}(\tau)= & \frac{2(2 \pi)^{1-\nu}}{\pi} k_{1} \int_{0}^{\infty} \frac{1-\cos (\omega \tau)}{\omega^{1+\nu}} \mathrm{d} \omega \\
= & 4(2 \pi)^{-\nu} k_{1} \int_{0}^{\infty} \frac{1-\cos (\omega \tau)}{\omega}\left(\frac{\omega^{1-\nu}}{1-\nu}\right)^{\prime} \mathrm{d} \omega \\
= & -4(2 \pi)^{-\nu} k_{1} \frac{1}{1-\nu} \\
& \times \int_{0}^{\infty} \frac{\tau \sin (\omega \tau)}{\omega^{\nu}} \mathrm{d} \omega+\frac{\sigma_{1}^{2}(\tau)}{1-\nu} \\
= & \frac{4(2 \pi)^{-\nu} k_{1}}{\nu} \sqrt{\pi} 2^{-\nu} \frac{\Gamma\left(1-\frac{\nu}{2}\right)}{\Gamma\left(\frac{1+\nu}{2}\right)}|\tau|^{\nu} \\
= & \frac{4 \gamma_{1} k_{1}}{\nu}|\tau|^{\nu}
\end{aligned}
$$

where

$$
\gamma_{1}=\frac{(2 \pi)^{-\nu} \sqrt{\pi} 2^{-\nu} \Gamma\left(1-\frac{\nu}{2}\right)}{\Gamma\left(\frac{1+\nu}{2}\right)}
$$

and $\Gamma(z)=\int_{0}^{\infty} t^{z-1} e^{-t} \mathrm{~d} t$. We note in passing that if the quantity $\nu$ is assumed sufficiently small, we can approximate by $\gamma_{1} \simeq 1$, so that the expression for the variogram of $\phi_{1}$ given in (25) can be approximated as

$$
\sigma_{1}^{2}(\tau)=\frac{4 k_{1}}{\nu}|\tau|^{\nu}
$$

Substitution of (25) in the autocorrelation of $w_{1}(t)$ yields

$$
\begin{aligned}
R_{w w_{1}}(\tau) & =e^{-\frac{2 \gamma_{1} k_{1}}{\nu}|\tau|^{\nu}} \\
& =\sum_{n=0}^{\infty} \frac{\left(-\frac{2 \gamma_{1} k_{1}}{\nu}|\tau|^{\nu}\right)^{n}}{n !} \\
& =1+\sum_{n=1}^{\infty} R_{w w_{1}, n}(\tau) .
\end{aligned}
$$

Based on (28), we can represent the PSD of the RP $w_{1}(t)$ through the infinite sum of sub-spectra $S_{w_{1}, n}(\omega)$, resulting from sub-terms of the autocorrelation function of the form

$$
R_{w w_{1}, n}(\tau)=\frac{\left(-\frac{2 \gamma_{1} k_{1}}{\nu}|\tau|^{\nu}\right)^{n}}{n !} .
$$

The sub-spectra $S_{w_{1}, n}(\omega)$ can be evaluated as

$$
\begin{aligned}
& S_{w_{1}, n}(\omega) \\
& =\frac{\left(-\frac{2 \gamma_{1} k_{1}}{\nu}\right)^{n}}{n !} \frac{2^{1+n \nu} \sqrt{\pi} \Gamma\left(\frac{1}{2}+\frac{n \nu}{2}\right)}{\Gamma\left(-\frac{n \nu}{2}\right)} \frac{1}{\left|\omega^{1+n \nu}\right|} \\
& =(-1)^{n+1} \frac{2 \sin \left(\frac{\pi n \nu}{2}\right) \nu\left(\frac{2 \gamma_{1} k 1}{\nu}\right)^{n} \Gamma(n \nu)}{\Gamma(n)} \frac{1}{\left|\omega^{1+n \nu}\right| .}
\end{aligned}
$$

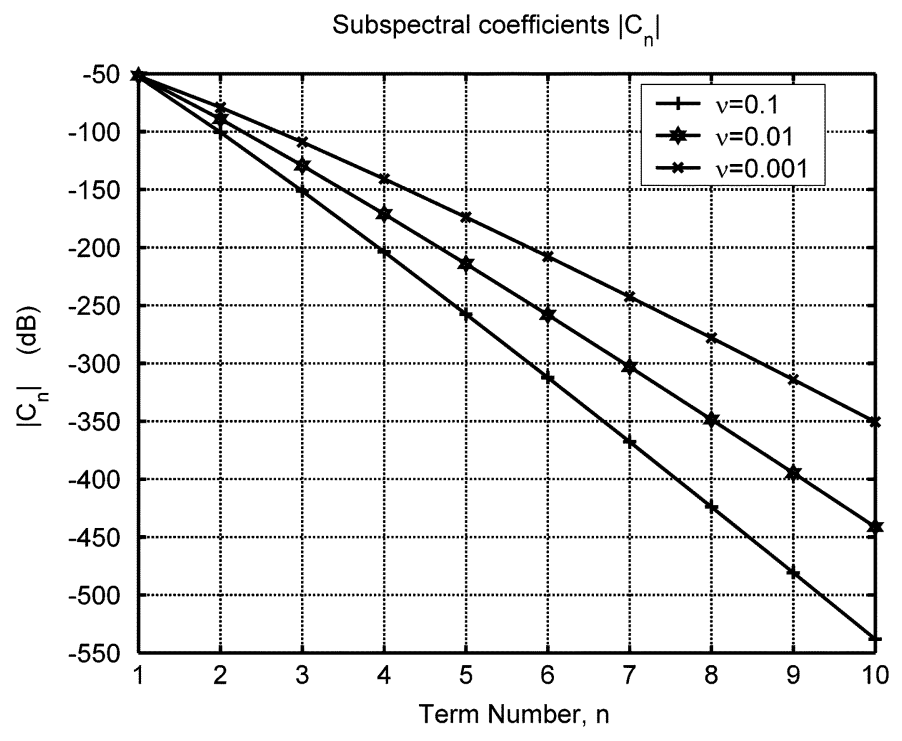

Fig. 3. Sub-spectral coefficients for $k_{1}=10^{-6}, \nu=0.1,0.01,0.001$.

For the derivation of (30), we have used the "Duplication Formula" for the $\Gamma$ function [25]

$$
\Gamma(2 z)=(2 \pi)^{-\frac{1}{2}} 2^{2 z-\frac{1}{2}} \Gamma(z) \Gamma\left(z+\frac{1}{2}\right)
$$

and the "Reflection Formula" [25]

$$
\Gamma(z) \Gamma(-z)=-\frac{\pi}{z \sin (\pi z)}
$$

The overall oscillator PSD is therefore

$$
\begin{aligned}
S_{w_{1}}(\omega) & =2 \pi \delta(\omega)+\sum_{n=1}^{\infty} S_{w_{1}, n}(\omega) \\
& =2 \pi \delta(\omega)+\sum_{n=1}^{\infty} C_{n}\left(\nu, k_{1}\right)\left|\omega^{-1-n \nu}\right| .
\end{aligned}
$$

We define as $C_{n}$ the coefficient of the $n$th sub-spectral term

$$
C_{n}\left(\nu, k_{1}\right)=(-1)^{n+1} \frac{2 \sin \left(\frac{\pi n \nu}{2}\right) \nu\left(\frac{2 \gamma_{1} k 1}{\nu}\right)^{n} \Gamma(n \nu)}{\Gamma(n)} .
$$

The convergence of the sub-spectra coefficients $C_{n}$ for large $n$ is ensured by the requirement $0<\nu<1$;

$$
\lim _{n \rightarrow \infty} C_{n}=0 .
$$

In Fig. 3 we plot the absolute value of the sub-spectra coefficients, $\left|C_{n}\right|$ versus $n$ for various values of $\nu$ and $k_{1}=10^{-6}$. We see that $C_{n}$ decreases rapidly with $n$ but the rate of decrease falls as $\nu$ approaches 0 . The dominant spectral component $\tilde{S}_{w_{1}}$, at frequencies $\omega \gg 0$ is the sub-spectral term for $n=1$

$$
\tilde{S}_{w_{1}}(\omega)=\frac{4 \sin \left(\frac{\pi \nu}{2}\right) \gamma_{1} k_{1} \Gamma(\nu)}{\left|\omega^{1+\nu}\right|} .
$$




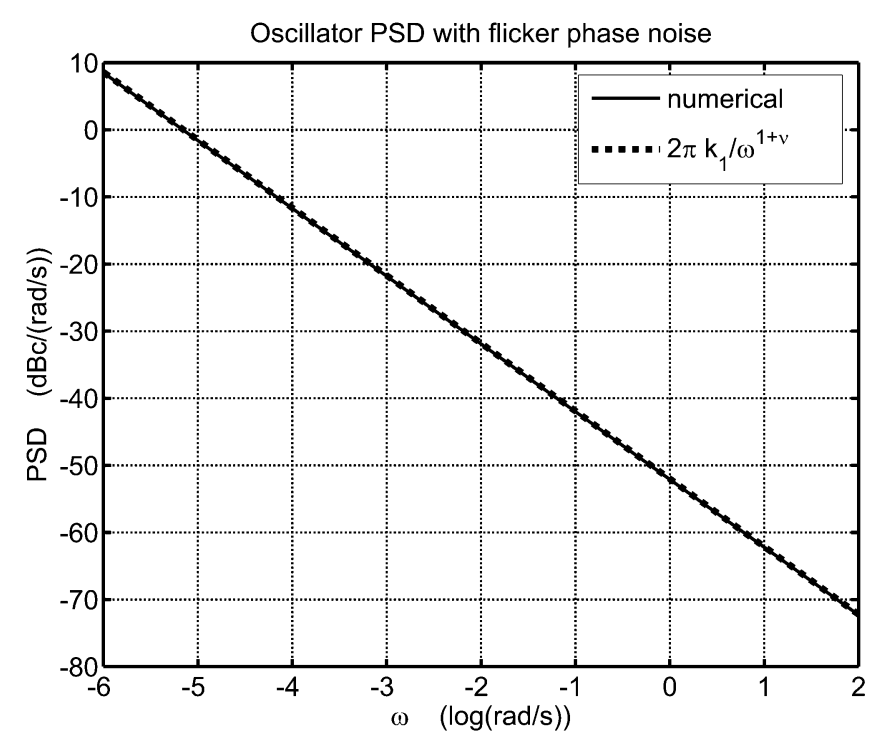

Fig. 4. Oscillator PSD with flicker phase noise $k_{1}=10^{-6}, \nu=0.01$.

For small values of $\nu$, we can algebraically approximate (34) using

$$
\lim _{\nu \rightarrow 0} 4 \sin \left(\frac{\pi \nu}{2}\right) \gamma_{1} \Gamma(\nu)=2 \pi
$$

so that, as we expected

$$
\tilde{S}_{w_{1}}(\omega)=\frac{2 \pi k_{1}}{\left|\omega^{1+\nu}\right|} .
$$

In Fig. 4, we plot the dominant spectral component $\tilde{S}_{w_{1}}(\omega)$ in (34) together with the numerically evaluated PSD given by the infinite sum in (31), for $\nu=0.01$ and $k_{1}=10^{-6}$. The graph demonstrates that the exact PSD matches extremely well the identified dominant spectral term $\tilde{S}_{w_{1}}(\omega)$ even for very small frequencies of the order of $10^{-6} \mathrm{rad} / \mathrm{s}$.

Focussing on frequencies close to the carrier, the behavior of the infinite sum in (31) for $\omega \ll 1$, stems from two opposing tendencies: as $\omega \rightarrow 0$ the quantity $\left|\omega^{-1-n \nu}\right| \rightarrow \infty$, at an increasing rate as $n \rightarrow \infty$. On the other hand, as noted above $C_{n} \rightarrow 0$ as $n \rightarrow \infty$. Those two opposing tendencies, suggest that an increasing number of terms in the infinite sum (31) have a non-negligible contribution to the close to the carrier PSD as $\omega \rightarrow 0$. The effect is depicted in Fig. 5, where we plot the absolute value of the sub-spectral terms, $\left|S_{w_{1}, n}\right|$, versus $n$ for various values of $\omega$.

To evaluate the oscillator PSD on the carrier frequency, we would need to perform the summation including all the terms in (31). Alternatively, we may integrate (28)

$$
\begin{aligned}
S_{w_{1}}(0) & =2 \int_{0}^{\infty} e^{-\frac{2 \gamma_{1} k_{1}}{\nu}|\tau|^{\nu}} \mathrm{d} \tau \\
& =8 \pi \frac{\Gamma\left(\frac{1}{\nu}\right)}{\nu} \sqrt[\nu]{-\frac{\Gamma\left(\frac{1+\nu}{2}\right)}{\sqrt{\pi} k_{1} \Gamma\left(-\frac{\nu}{2}\right)}} .
\end{aligned}
$$

Thus, in (31) the first term, $2 \pi \delta(\omega)$, combines with the infinite sum in the second term to give the finite value given in (36). The finite PSD at dc along with stationarity of the oscillator, implied

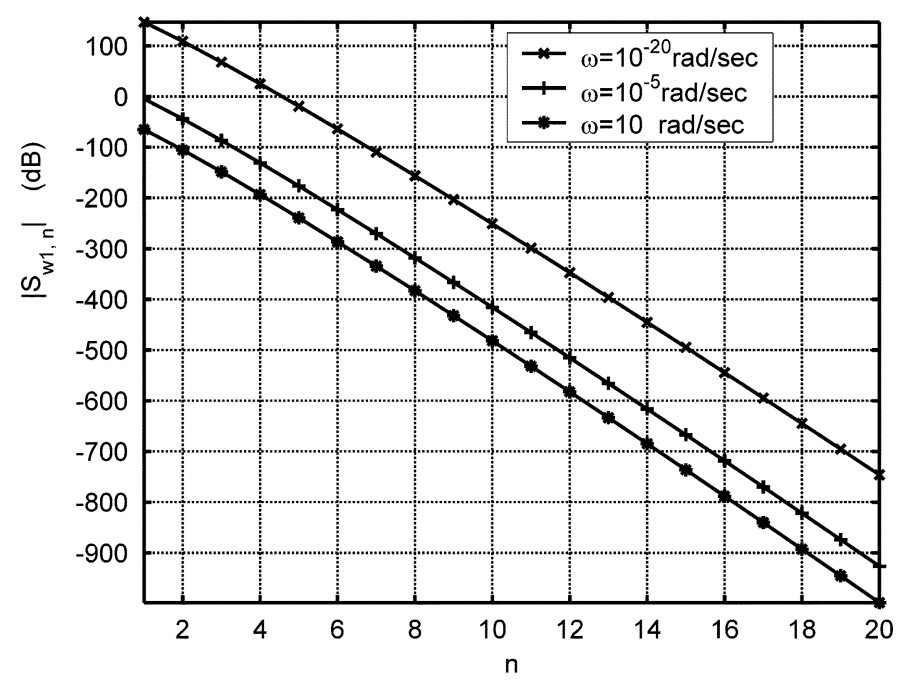

Fig. 5. $\left|S_{w_{1}, n}\right|$ versus $n$ for flicker phase noise $k_{1}=10^{-6}, \nu=0.01$.

TABLE II

$S_{w_{1}}(0)$ OF $w_{1}(t)$ FOR $k_{1}=10^{-6}$

\begin{tabular}{cc}
\hline$\nu$ & $S_{w_{1}}(0)$ \\
\hline \hline 0.1 & $0.2596 \cdot 10^{55}$ \\
0.01 & $0.5216 \cdot 10^{529}$ \\
0.001 & $0.2650 \cdot 10^{5268}$ \\
\hline
\end{tabular}

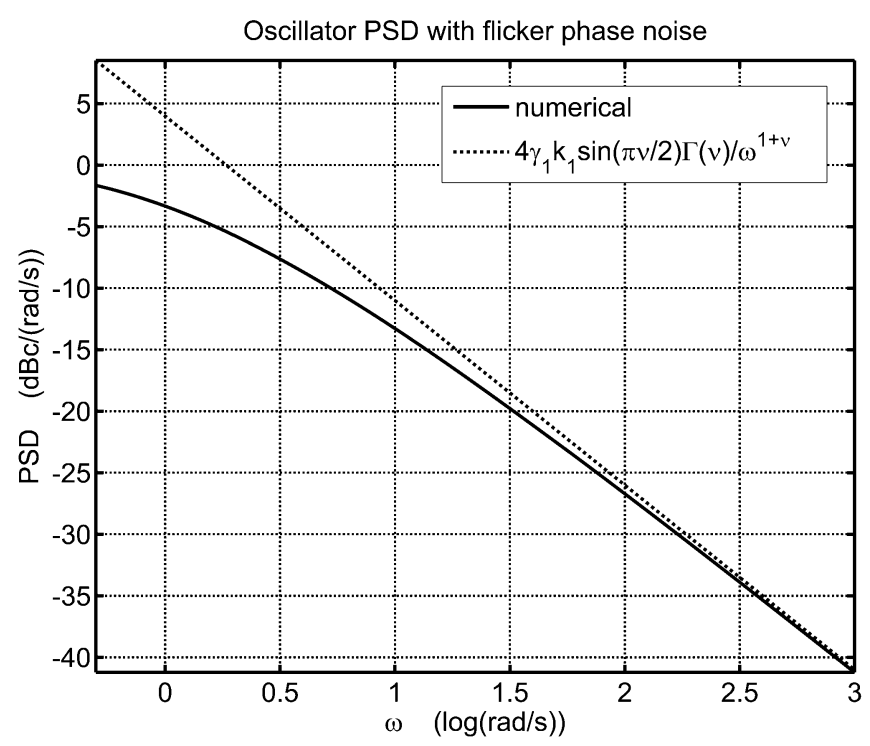

Fig. 6. Oscillator PSD with flicker phase noise $k_{1}=1, \nu=0.5$.

by $R_{w w_{1}}(0)=1$ and $\langle w(t)\rangle=0$, are two very important conclusions of the present analysis, shedding light on the question of "infrared catastrophe" in oscillatory systems with pink phase noise. In Table II, we have evaluated the carrier PSD $S_{w_{1}}(0)$ of the RP $w_{1}(t)$ for various values of $\nu$ and $k_{1}=10^{-6}$.

An immediate conclusion of the above is that the PSD of the $\mathrm{RP} w_{1}(t)$ can be expressed as $2 \pi k_{1}\left|\omega^{-1-\nu}\right|$ at frequencies away from the carrier while near dc curves to approach a finite (although very large for small values of $\nu$ ) value, expressed in (36). To illustrate the effect, in Fig. 6, we plot the numerically evaluated oscillator PSD around the oscillation frequency, for $k_{1}=1$ 
and $\nu=0.5$. The oscillator PSD is evaluated through (36) at $0 \mathrm{~dB}$. In the graph is depicted how the oscillator PSD at small frequencies approaches at a slowly increasing rate the value of $S_{w_{1}}(0)$.

To summarize the findings of this section, $1 /|f|^{1+\nu}$, $0<\nu \ll 1$ phase noise results in a WSS noise component $w_{1}(t)$. The oscillator follows a $2 \pi k_{1}\left|\omega^{-1-\nu}\right|$ characteristic at high frequencies, while it approaches a finite PSD on the carrier frequency, equal to $8 \pi\left(\Gamma((1 / \nu)) /(\nu) \sqrt[\nu]{-\left(\Gamma((1+\nu) /(2)) /\left(\sqrt{\pi} k_{1} \Gamma(-(\nu) /(2))\right.\right.}\right.$.

\section{WhITE FM PHASE NOISE}

The case of white frequency modulated phase noise has been already addressed in the literature and detailed approaches exist both in the frequency and time domain [10], [3]. For the sake of completeness it is briefly covered in the present section.

Let us assume phase noise $\phi_{2}(t)$ that is generated by white frequency noise with PSD

$$
S_{\Omega_{2}}(\omega)=4 \pi^{2} k_{2}
$$

The variance $\sigma_{2}^{2}(\tau)$ of the phase noise variation $\mathrm{RP} \xi_{2}(t, \tau)=$ $\phi_{2}(t+\tau)-\phi_{2}(t)$ obtained from (15) is

$$
\sigma_{2}^{2}(\tau)=8 \pi k_{2} \int_{0}^{\infty} \frac{1-\cos (\omega \tau)}{\omega^{2}} \mathrm{~d} \omega=4 \pi^{2} k_{2}|\tau| .
$$

The autocorrelation function and the PSD of the RP $w_{2}(t)=$ $e^{j \phi_{2}(t)}$ are consequently evaluated to be

$$
\begin{aligned}
R_{w w_{2}}(\tau) & =e^{-2 \pi^{2} k_{2}|\tau|} \\
S_{w_{2}}(\omega) & =\frac{4 \pi^{2} k_{2}}{4 \pi^{4} k_{2}^{2}+\omega^{2}} .
\end{aligned}
$$

The PSD of the RP $w_{2}(t)$ is a Lorentzian and the resulting oscillator process $\psi_{2}(t)=e^{j\left(\omega_{\mathrm{osc}} t+\phi_{2}(t)\right)}$ is stationary. Power conservation is confirmed as

$$
R_{w w_{2}}(0)=\frac{1}{2 \pi} \int_{-\infty}^{\infty} S_{w_{2}}(\omega) \mathrm{d} \omega=1
$$

For $\omega \gg 2 \pi^{2} k_{2}$ the PSD can be approximated as

$$
S_{w_{2}}(\omega) \simeq \frac{4 \pi^{2} k_{2}}{\omega^{2}}
$$

\section{FLICKER FM PHASE NOISE}

The case of $\alpha=3$ has been examined by Klimovitch in [12] and [13]. We briefly repeat these results using a notation consistent with that in the rest of the paper.

Let us assume phase noise $\phi_{3}(t)$ that is generated by frequency fluctuations having a flicker noise PSD as following:

$$
S_{\Omega_{3}}(\omega)=\frac{8 \pi^{3}(2 \pi)^{-\nu} k_{3}}{\left|\omega^{1-\nu}\right|}
$$

where $\nu \rightarrow 0$.
To derive the PSD of the process $w_{3}(t)=e^{j \phi_{3}(t)}$ we need to evaluate the variance $\sigma_{3}^{2}(\tau)$ of the phase noise variation $\xi_{3}(\tau)=$ $\phi_{3}(t+\tau)-\phi_{3}(t)$

$$
\begin{aligned}
\sigma_{3}^{2}(\tau) & =16 \pi^{2}(2 \pi)^{-\nu} k_{3} \int_{0}^{\infty} \frac{1-\cos (\omega \tau)}{\omega^{3-\nu}} \mathrm{d} \omega \\
& =K|\tau|^{2-\nu}
\end{aligned}
$$

where

$$
K=\frac{16 \pi^{2} \sqrt{\pi}(2 \pi)^{-\nu} \Gamma\left(\frac{\nu}{2}\right) k_{3}}{(2-\nu) \Gamma\left(\frac{3}{2}-\frac{\nu}{2}\right)} .
$$

By series expansion of $R_{w w}(\tau)=e^{-\left(K|\tau|^{2-\nu}\right) /(2)}$ around $\nu=0$ and keeping only first-order terms, we can express the autocorrelation function $R_{w w_{3}}(\tau)$ of $w_{3}(t)$ as

$$
\begin{aligned}
R_{w w_{3}}(\tau) & =e^{-\frac{\sigma_{3}^{2}(\tau)}{2}} \\
& \simeq e^{-\frac{K|\tau|^{2}}{2}}+\frac{\nu}{2} K e^{-\frac{K|\tau|^{2}}{2}}|\tau|^{2} \ln (|\tau|)
\end{aligned}
$$

and the PSD of $w_{3}(t)$

$$
S_{w_{3}}(\omega)=\frac{\sqrt{2 \pi}}{\sqrt{K}} e^{-\frac{\omega^{2}}{2 K}}+\mathcal{F}\left[\frac{\nu}{2} K e^{-\frac{K|\tau|^{2}}{2}}|\tau|^{2} \ln (|\tau|)\right] .
$$

Numerical results presented in [12] and [13] lead to the conclusion that close to the carrier, the dominant term in (46) is the Gaussian part $\sqrt{2 \pi} \sqrt{K^{-1}} e^{-\left(\omega^{2}\right) /(2 K)}$ while $\mathcal{F}\left[(\nu / 2) K e^{-\left(K|\tau|^{2}\right) /(2)}|\tau|^{2} \ln (|\tau|)\right]$ is proportional to $|\omega|^{-3}$ at frequencies where the small-angle approximation is valid.

As $\nu \rightarrow 0, S_{w_{3}}(\omega)$ is approximately Gaussian over a larger range of frequencies. This result is in agreement with the theory of long correlated RPs, as described in [10]. However it is worth noting that the case of $\nu=0$ is similar to the corresponding case in the analysis of flicker phase noise. For $\nu=0$ the RP $w_{3}(t)$ becomes nonstationary with $R_{w w_{3}}(\tau)=0, \tau \neq 0$. This result follows intuitively as $1 /|f|^{3}$ phase noise is expected to be generated by frequency modulated nonstationary flicker noise.

\section{RANDOM WALK FM PHASE NOISE}

Close to the oscillator frequency $\omega_{\text {osc }}$, measurements have shown that the PSD follows a $1 / f^{4}$ characteristic [26]. This kind of noise can be considered to be frequency modulated Brownian noise. In other words, the generation mechanism involves frequency modulation of a Lorentzian-type PSD.

We consider random walk FM noise $\phi_{4}(t)$ generated by Brownian frequency noise $\Omega_{4}(t)$ of PSD

$$
S_{\Omega_{4}}(\omega)=\frac{16 \pi^{4} k_{4}}{\rho^{2}+\omega^{2}} .
$$

The variance $\sigma_{4}^{2}(\tau)$ of the phase noise variation $\mathrm{RP} \xi_{4}(t, \tau)=$ $\phi_{4}(t+\tau)-\phi_{4}(t)$ is derived as follows:

$$
\begin{aligned}
\sigma_{4}^{2}(\tau) & =32 \pi^{3} k_{4} \int_{0}^{\infty} \frac{1-\cos (\omega \tau)}{\omega^{2}} \frac{1}{\rho^{2}+\omega^{2}} \mathrm{~d} \omega \\
& =\frac{16 \pi^{4} k_{4}}{\rho^{3}}\left(-1+\rho|\tau|+e^{-\rho|\tau|}\right) .
\end{aligned}
$$


The autocorrelation function of the $\mathrm{RP} w_{4}(t)=e^{j \phi_{4}(t)}$ is therefore

$$
R_{w w_{4}}(\tau)=e^{\frac{8 \pi^{4} k_{4}}{\rho^{3}}\left(1-\rho|\tau|-e^{-\rho|\tau|}\right)}
$$

and the PSD of $w_{4}(t)$ is evaluated as the $\mathcal{F}$ of (49).

The exact PSD of $w_{4}(t)$ can be obtained by convolving the Lorentzian PSD $\left(16 \pi^{4} \rho^{2} k_{4}\right) /\left(64 \pi^{8} k_{4}^{2}+\rho^{4} \omega^{2}\right)$ with $\mathcal{F}\left[e^{\left(8 \pi^{4} k_{4}\right) /\left(\rho^{3}\right)\left(1-e^{-\rho|\tau|}\right)}\right]$. Unfortunately, no closed form expression can be obtained for $\mathcal{F}\left[e^{\left(8 \pi^{4} k_{4}\right) /\left(\rho^{3}\right)\left(1-e^{-\rho|\tau|}\right)}\right]$.

Alternatively, as $R_{w w 4}(\tau)$ is a rapidly decreasing function of $\tau$, an approximation for $R_{w w_{4}}(\tau)$ can be derived by taking the Taylor series of $\sigma_{4}^{2}(\tau)$ around $\tau=0$ and keeping only the first nonvanishing terms

$$
R_{w w_{4}}(\tau) \simeq \tilde{R}_{w w_{4}}(\tau) \doteq e^{-\frac{4 \pi^{4} k_{4}}{\rho}|\tau|^{2}} .
$$

The PSD of the approximate autocorrelation function $\tilde{R}_{w w_{4}}(\tau)$ is Gaussian (bell-shaped) and can be expressed as

$$
\tilde{S}_{w_{4}}(\omega)=\frac{\sqrt{\rho}}{2 \pi \sqrt{\pi k_{4}}} e^{-\frac{\rho \omega^{2}}{16 \pi^{4} k_{4}}} .
$$

The approximate PSD in (51) fails at frequencies $\omega \gg 0$ as an increasing number of terms in the series expansion of $\sigma_{4}^{2}(\tau)$ will have a non-negligible effect. However, the conclusions of the small-angle approximation are applicable in that frequency region. (51) in conjunction with the small-angle approximation suggest that the PSD of $w_{4}(t)$ can be expressed as

$$
S_{w_{4}}(\omega)=\tilde{S}_{w_{4}}(\omega)+\frac{\mu}{\omega^{4}} u\left(|\omega|-\omega_{4}\right)
$$

where $u(\cdot)$ is the step function and $\omega_{4}$ is the frequency of transition from the Gaussian to the power-law characteristic $\mu w^{-4}$. The small-angle approximation dictates that

$$
\mu=16 \pi^{4} k_{4} .
$$

The unknowns $\omega_{4}$ and $\rho$ may be determined from: i) the overall power of $w_{4}(t)$ is unity since $R_{w w_{4}}(0)=1$; and ii) the PSD is a continuous function so that at $\omega_{4}$ the Gaussian and the power law characteristic equate. These two conditions yield the following system of equations:

$$
\left\{\begin{array}{l}
\int_{0}^{\omega_{4}} \tilde{S}_{w_{4}}(\omega) \mathrm{d} \omega+\int_{\omega_{4}}^{\infty} \frac{16 \pi^{4} k_{4}}{\omega^{4}} \mathrm{~d} \omega=\pi \\
\tilde{S}_{w_{4}}\left(\omega_{4}\right)=\frac{16 \pi^{4} k_{4}}{\omega_{4}^{4}}
\end{array}\right.
$$

which is equivalent to

$$
\left\{\begin{array}{l}
\pi \operatorname{erf}\left(\frac{\rho \omega_{4}}{4 \pi^{2} \sqrt{k 4}}\right)+\frac{16 \pi^{4} k_{4}}{3 \omega_{4}^{3}}=\pi \\
\omega_{4}=\frac{4 \pi^{2}}{\rho} \sqrt{-2 \rho k_{4} \mathrm{~W}_{[-1]}\left(-\frac{\sqrt{2 \rho \sqrt{\frac{\rho}{\pi k_{4}}}}}{8 \pi}\right)}
\end{array}\right.
$$

where $\operatorname{erf}(\cdot)$ denotes the error function and $\mathrm{W}_{[-1]}(\cdot)$ the -1 branch of the Lambert-W function [27] that satisfies the relation

$$
\mathrm{W}(x) e^{\mathrm{W}(x)}=x
$$

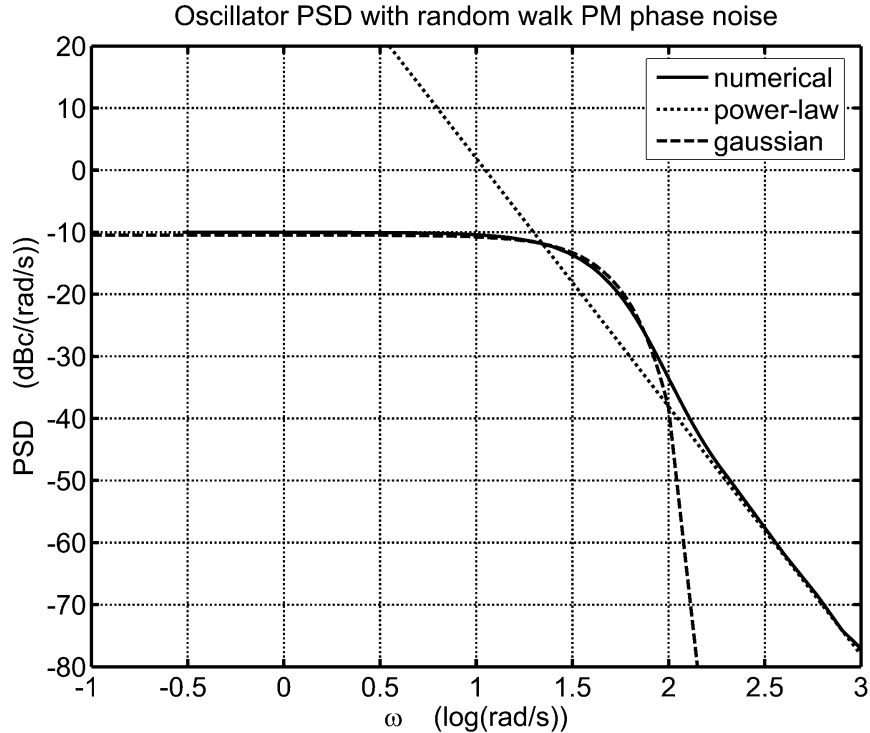

Fig. 7. Oscillator PSD with $1 / f^{4}$ phase noise. $k_{4}=10, \rho=10$.

so that we evaluate the larger of the two real and positive roots on $\omega_{4}$.

In Fig. 7, we plot the numerically evaluated $\mathcal{F}\left[R_{w w 4}(\tau)\right]$ from (49) for $k_{4}=10$ and $\rho=10$. On the same graph are illustrated the Gaussian part of the PSD and the power-law region for the same values of $k_{4}$ and $\rho$. By substitution in (55), the knee frequency is evaluated $\omega_{4}=99.317 \mathrm{rad} / \mathrm{s}$ and provides a good approximation to the knee frequency depicted in the graph, $\omega_{4}=99.266 \mathrm{rad} / \mathrm{s}$. This demonstrates that $S_{w_{4}}(\omega)$ matches reasonably well with the numerically evaluated PSD of $w_{4}(t)$ as long as $R_{w w_{4}}(\tau)$ can be considered a rapidly decreasing function of $\tau$.

Concluding the discussion on $1 / f^{4}$ noise, the PSD of an oscillator with frequency modulated Brownian noise can be well approximated by a Gaussian PSD below the transitional frequency $\omega_{4}$ and then exhibits a $16 \pi^{4} k_{4} \omega^{-4}$ PSD in accordance with the small-angle approximation.

\section{ENhanced Oscillator Spectral Model}

Combining the results of Sections III to VII, we propose an enhanced oscillator model based on the following observations.

1) Phase noise can be well approximated as the sum of power-law processes [4], [5] resulting from integration of frequency fluctuations [6], [10].

2) Band-limited white-like phase noise is expected to result in a carrier component added to a band-limited white-like noise region, (21).

3) Flicker phase noise of $1 /|f|^{1+\nu}$ PSD results in a finite variance noise process $e^{j \phi_{1}(t)}$. The dominant side-band spectral component follows a $2 \pi k_{1}\left|\omega^{-1-\nu}\right|$ characteristic, while the PSD on the carrier frequency is finite and expressed as $S_{w_{1}}(0)=8 \pi \frac{\Gamma\left(\frac{1}{\nu}\right)}{\nu} \sqrt[\nu]{-\frac{\Gamma\left(\frac{1+\nu}{2}\right)}{\sqrt{\pi} k_{1} \Gamma\left(-\frac{\nu}{2}\right)}},(36)$.

4) In the general case, the oscillator output is a nonstationary process [6]. However, the analysis presented implies that an oscillator with power-law phase noise as discussed can 
be treated as a WSS RP. In that context, the following are true.

- At relatively high frequencies where the small-angle approximation is valid the oscillator PSD coincides with the phase noise PSD.

- Long correlated events in the oscillator phase will generate Gaussian-like components in the oscillator PSD. $1 /|f|^{3}$ and $1 / f^{4}$ phase noise are such processes, (46) and (52).

- Short correlated events in the oscillator phase give rise to Lorentzian-like components in the oscillator PSD. $1 / f^{2}$ phase noise is such a process, (40).

- Long correlated events dominate on the near-carrier regime.

- The overall oscillator power of a unit amplitude complex valued oscillator is unity.

Based on the previous remarks, the ambiguity about the nearcarrier regime can be lifted and an approximate model can be derived. Starting with phase noise resulting from the addition of uncorrelated power-law phase noise processes;

$$
\phi(t)=\phi_{0}(t)+\phi_{1}(t)+\phi_{2}(t)+\phi_{3}(t)+\phi_{4}(t)
$$

we deduce that

$$
w(t)=w_{0}(t) \cdot w_{1}(t) \cdot w_{2}(t) \cdot w_{3}(t) \cdot w_{4}(t) .
$$

Since the processes $\phi_{\alpha}(t), \alpha \in\{0,1,2,3,4\}$ are uncorrelated, the PSD of the process $w(t)$ can be expressed as

$$
\begin{array}{r}
S_{w}(\omega)=S_{w_{0}}(\omega) \otimes S_{w_{1}}(\omega) \otimes S_{w_{2}}(\omega) \\
\quad \otimes S_{w_{3}}(\omega) \otimes S_{w_{4}}(\omega)
\end{array}
$$

where $\otimes$ denotes convolution. At frequencies where the smallangle approximation is applicable we can however express the PSD as

$$
\begin{aligned}
e^{j \phi(t)} \simeq & 1+j \phi(t) \Rightarrow \\
S_{w}(\omega) \simeq & 2 \pi \delta(\omega)+S_{w_{0}}(\omega)+S_{w_{1}}(\omega) \\
& +S_{w_{2}}(\omega)+S_{w_{3}}(\omega)+S_{w_{4}}(\omega) .
\end{aligned}
$$

At frequencies away from the oscillation frequency, (59) indicates that the overall oscillator PSD can be expressed as the sum of the power-law phase noise processes sub-spectra. On the other hand, close to the carrier where the small-angle approximation fails, the oscillator PSD must be evaluated by the convolution of the relevant near-carrier sub-spectra.

From the preceding analysis we deduce that the near-carrier PSD is expressed as the convolution of a Dirac delta generated by purple frequency noise components, a very high and narrow region resulting from flicker phase noise sources, a narrow Lorentzian region and two Gaussian regions. The convolution of the two Gaussian regions with the Dirac delta and the flicker noise PSD generates a Gaussian region. Further convolution with the Lorentzian, produces the overall PSD regime in the near-carrier frequency region. In that context, based on the fact that measurements typically find that $k_{2} \ll k_{4}$, we expect the resulting Gaussian region to be notably wider than the

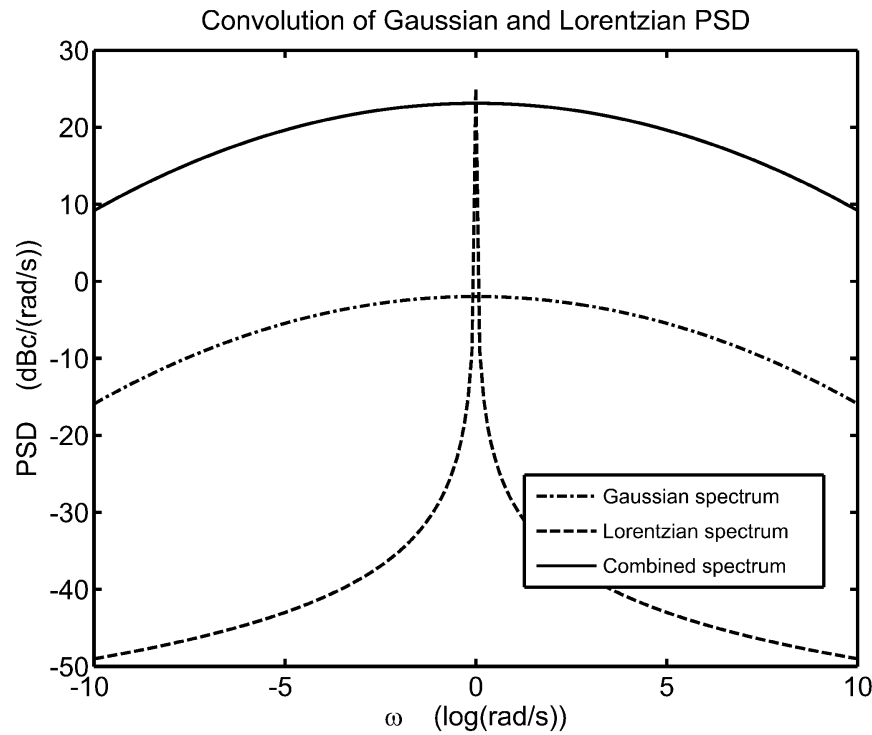

Fig. 8. Convolution of Gaussian and Lorentzian PSD.

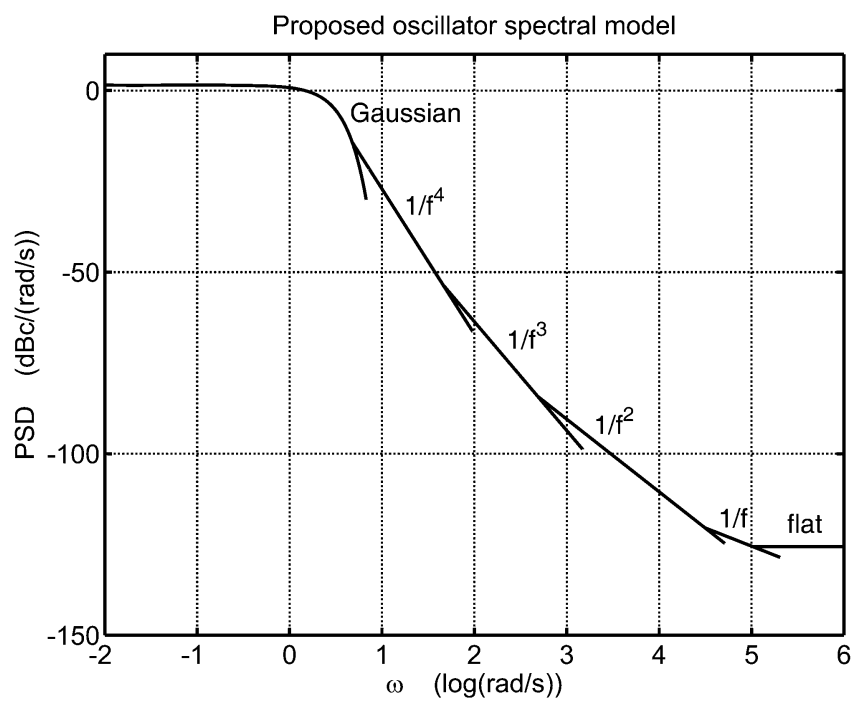

Fig. 9. Oscillator PSD with Gaussian characteristic near the oscillator frequency.

Lorentzian. Consequently, their convolution will result in an approximately Gaussian PSD. The effect is illustrated in Fig. 8, for $k_{2}=10^{-4}, k_{4}=1$ and $\rho=50\left(\sigma^{2}=15.5855\right)$.

Concluding this discussion, we propose an enhanced oscillator model including:

1) a Gaussian-like region near the carrier frequency, as an approximation of the convolution of the near-carrier subspectra;

2) a sequence of power-law regions, in accordance with the small-angle approximation.

The model is depicted in Fig. 9 and assumes that the oscillator can be treated as a WSS RP and that flicker phase noise sources generate finite variance noise terms.

We can readily evaluate the Gaussian region variance and the lower corner frequency $\omega_{4}$ of the $1 / f^{4}$ region by following a similar analysis to the one presented in Section VII, the overall 
variance of the RP $w(t)$ is unity, while the Gaussian and $1 / f^{4}$ characteristics equate at $\omega_{4}$. Expressions (60) to (65) provide the basis for the evaluation of the oscillator PSD at any $\omega$ under the proposed enhanced oscillator model. The angular frequencies $\omega_{0}, \omega_{1}, \omega_{2}, \omega_{3}$ and $\omega_{4}$ that represent the knee frequencies between the flat and $1 / f$ region, the $1 / f$ and $1 / f^{2}, 1 / f^{2}$ and $1 / f^{3}, 1 / f^{3}$ and $1 / f^{4}, 1 / f^{4}$ and Gaussian regions respectively, are evaluated by equating the relevant power spectral densities at these points.

$$
\begin{aligned}
\omega_{0}= & \frac{2 \pi k_{1}}{k_{0}} \\
\omega_{1}= & \frac{2 \pi k_{2}}{k_{1}} \\
\omega_{2}= & \frac{2 \pi k_{3}}{k_{2}} \\
\omega_{3}= & \frac{2 \pi k_{4}}{k_{3}} \\
\frac{1}{\sqrt{2 \pi} \sigma} e^{-\frac{\omega_{4}^{2}}{2 \sigma^{2}}=} & \frac{16 \pi^{4} k_{4}}{\omega_{4}^{4}} \\
\frac{1}{2} & \operatorname{erf}\left(\frac{\omega_{4}}{\sqrt{2} \sigma}\right)+\frac{16 \pi^{4} k_{4}\left(\omega_{3}^{3}-\omega_{4}^{3}\right)}{3 \omega_{4}^{3} \omega_{3}^{3}} \\
& +\frac{4 \pi^{3} k_{3}\left(\omega_{2}^{2}-\omega_{3}^{2}\right)}{\omega_{2}^{2} \omega_{3}^{2}}+\frac{4 \pi^{2} k_{2}\left(\omega_{1}-\omega_{2}\right)}{\omega_{1} \omega_{2}} \\
& +2 \pi k_{1}\left(\ln \left(\omega_{0}\right)-\ln \left(\omega_{1}\right)\right) \\
& +k_{0}\left(\omega_{B}-\omega_{0}\right) \\
= & \pi
\end{aligned}
$$

Finally, $\omega_{B}$ denotes the cutoff frequency of the white region, generally determined by the low-pass filter characteristics of the oscillator system.

\section{CONCLUSION}

In this paper, we have used correlation theory methods to evaluate the near-carrier PSD for the power-law phase noise processes present in oscillators. We have shown that even in the near-carrier region it is possible to obtain accurate approximations for the oscillator PSD that result from power-law processes. We have presented new theoretical analyses of the nearcarrier PSD for three important cases; purple, blue and brown frequency noise. An interesting consequence of our analysis is that stationarity of the oscillator output is not destroyed by approximately flicker phase noise.

We have derived an enhanced model of the oscillator PSD by combining the noise spectra arising from constituent power-law processes. The parameters of this model are readily obtained from measurements. The model follows a Gaussian PSD at near-carrier frequencies followed by a sequence of power-law regions.

\section{ACKNOWLEDGMENT}

The authors would like to thank the anonymous reviewers for their valuable comments.

\section{REFERENCES}

[1] D. B. Leeson, "A simple model of feedback oscillator noise spectrum," Proc IEEE, vol. 54, no. 2, pp. 329-330, Feb. 1966.
[2] A. Hajimiri and T. Lee, "A general theory of phase noise in electrical oscillators," IEEE J. Solid-State Circuits, vol. 33, no. 2, pp. 179-194, Feb. 1998.

[3] A. Demir, A. Mehrotra, and J. Roychowdhury, "Phase noise in oscillators: A unifying theory and numerical methods for characterization," IEEE Trans. Circuits Syst. I, Fundam. Theory Appl., vol. 47, no. 5, pp. 655-674, May 2000.

[4] D. Allan, H. Hellwig, P. Kartaschoff, J. Vanier, J. Vig, G. Winkler, and N. Yannoni, "Standard terminology for fundamental frequency and time metrology," in Proc 42nd Ann. Freq. Contr. Symp., Jun. 1988, pp. 419-425.

[5] International Radio Consultative Committee C.C.I.R, Characterization of frequency and phase noise Geneva, Switzerland, 1986, Rep. 580, pp. $142-150$.

[6] J. A. Barnes, A. R. Chi, L. Cutler, D. Healey, D. Leeson, T. McGunigal, J. W. S. Mullen, Jr., R. Sydnor, R. Vessot, and G. Winkler, "Characterization of frequency stability," IEEE Trans. Instrum. Meas., vol. 20, no. 2, pp. 105-120, May 1971.

[7] J. A. Barnes and S. Jarvis, Jr., Efficient numerical and analog modelling of flicker noise processes (in U.S. Department of Commerce, National Bureau of Standards), Jun. 1971, Tech. Rep., NBS Technical Note 604.

[8] L. Bartosch, "Generation of colored noise," Int. J. Mod. Phys. C, vol. 12, no. 6, pp. 851-855, 2001.

[9] R. B. Staszewski, C. Fernando, and P. T. Balsara, "Event-driven simulation and modeling of phase noise of an RF oscillator," IEEE Trans. Circuits Syst. I, Fundam. Theory Appl., vol. 52, no. 4, pp. 723-733, Apr. 2005.

[10] S. Rytov, Principles of Statistical Radiophysics 2: Correlation Theory of Random Processes. New York: Springer, 1988, vol. 2.

[11] J. A. Mullen and D. Middleon, "Limiting forms of FM noise spectra," Proc. IRE, vol. 45, pp. 874-877, Jun. 1957.

[12] G. Klimovitch, "A nonlinear theory of near-carrier phase noise in freerunning oscillators," in Proc IEEE Intl Conf. Circuits Syst., Mar. 2000, vol. T80, pp. 1-6.

[13] _ _ "Near-carrier oscillator spectrum due to flicker and white noise," in Proc IEEE Intl Symp on Circuits and Systems, Geneva, Switzerland, May 2000, vol. I, pp. 703-706.

[14] A. Papoulis and S. U. Pillai, Probability, Random Variables and Stochastic Processes. New York: McGraw-Hill, 2002.

[15] D. Jupp, A. Strahler, and C. Woodcock, "Autocorrelation and regularization in digital images. I. Basic theory," IEEE Trans Geosci. Remote Sensing, vol. 26, no. 4, pp. 463-473, Jul. 1988.

[16] A. Papoulis, The Fourier Integral and its Applications. New York: McGraw-Hill, 1962.

[17] F. N. Hooge, "1/ $f$ noise sources," IEEE Trans. Electron. Devices, vol. 41, no. 11, pp. 1926-1935, Nov. 1994.

[18] A. Demir, "Phase noise and timing jitter in oscillators with colorednoise sources," IEEE Trans. Circuits Syst. I, Fundam. Theory Appl., vol. 49, no. 12, pp. 1782-1791, Dec. 2002.

[19] M. S. Keshner, "Renewal Process and Diffusion Models of 1/f Noise," Ph.D. dissertation, Elect. Eng. Comp. Sci. Dep., Massachusetts Institute of Technology, Cambridge, May 1979.

[20] _ _ "1/f noise," in Proc. IEEE, Mar. 1982, vol. 70, no. 3, pp. 212-218.

[21] B. B. Mandelbrot, Multifractals and 1/F Noise: Wild Self-Affinity in Physics (1963-1976): Selecta Volume N. New York: Springer-Verlag, Jan. 1999.

[22] P. H. Handel, "Fundamental quantum $1 / f$ noise in semiconductor devices," IEEE Trans. Electron. Devices, vol. 41, no. 11, pp. 2023-2033, Nov. 1994.

[23] P. Handel, " $1 / \mathrm{f}$ noise universality in high-technology applications," in Proc IEEE Int.Freq. Contr. Symp., Jun. 1994, pp. 8-21.

[24] P. H. Handel and C. S. Tsai, , P. Handel and A. Chung, Eds., "Quantum 1/f effect in $\mathrm{LiNbO}_{3}$ crystals," in Proceedings of the Symposium on Quantum $1 / f$ Noise and Other Low Frequency Fluctuations in Electronic Devices. St. Louis, MO: American Institute of Physics, Aug. 1998, pp. 156-161.

[25] M. Abramowicz and I. A. Stegun, Handbook of Mathematical Functions With Formulas, Graphs and Mathematical Tables. New York: Dover, 1974.

[26] I. Zamek and S. Zamek, "Crystal oscillators jitter measurements and its estimation of phase noise," in Proc. IEEE Int. Freq. Contr. Symp., May 2003, pp. 547-555.

[27] F. Chapeau-Blondeau and A. Monir, "Numerical evaluation of the Lambert W function and application to generation of generalized Gaussian noise with exponent 1/2," in IEEE Trans. Signal Process., Sep. 2002, vol. 50, no. 9, pp. 2160-2165. 


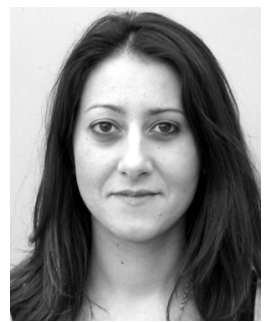

Arsenia Chorti received the M. Eng. degree in electrical engineering from the University of Patras, $\mathrm{Pa}$ tras, Greece, in 1998, the D.E.A. degree in electronics from the University Pierre et Marie Curie-Paris VI, France, in 2000 and the Ph.D. degree in communications and signal processing from Imperial College London, London,U.K., in 2005.

She is currently working as a Research Fellow in the Electronic Systems Design Group, Electronics and Computer Science Department, University of Southampton, Southampton, U.K., in the area of intelligent sensing. Her research interests include multicarrier communication systems, orthogonal frequency-division multiplexing (OFDM), stochastic signal processing, density estimation, novelty detection, and prediction/estimation algorithms.

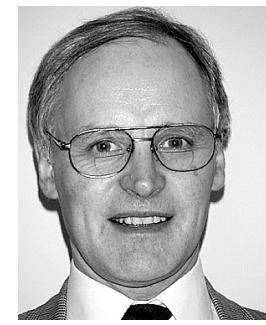

Mike Brookes (M'88) received the B.A. degree in mathematics from Cambridge University, Cambridge U.K. in 1972

Following this, he went to the U.S. where he spent four years at the Massachusetts Institute of Technology, Cambridge, working on astronomical instrumentation and telescope control systems. Since 1979, he has worked in the Electrical and Electronic Engineering Department, Imperial College London, London, U.K., where he is a Reader in the Communications and Signal Processing Research Group. His main areas of research are speech processing, and he has worked on speech production modeling, speaker recognition algorithms, and techniques for speech enhancement using both single microphones and microphone arrays. $\mathrm{He}$ is currently applying techniques from speech processing to RADAR target identification and is also actively involved in computer vision research. 